\title{
Clinical presentation, treatment modalities and outcome in patients with adrenocortical carcinoma: A single center experience
}

\author{
M. BRONSWIJK ${ }^{1}$, A. LAENEN ${ }^{2}$, O. E. BECHTER ${ }^{1, *}$ \\ ${ }^{1}$ Department of General Medical Oncology, University Hospitals Leuven, Campus Gasthuisberg, KU Leuven, Leuven, Belgium; ${ }^{2}$ Leuven Biosta- \\ tistics and Statistical Bioinformatics Centre, Leuven Cancer Institute, KU Leuven, Leuven, Belgium \\ ${ }^{*}$ Correspondence: Oliver.Bechter@uzleuven.be
}

Received January 5, 2019 / Accepted July 10, 2019

\begin{abstract}
Adrenocortical carcinoma is an orphan disease usually associated with a poor prognosis. Surgery is the only treatment with a curative intent, leaving systemic therapy mainly for the purpose of symptom control. First line combination chemotherapy with Etoposide, Doxorubicin, Cisplatin and Mitotane (EDP-Mitotane) is considered the standard of care, although this regimen is not associated with an overall survival benefit. Due to the rarity of the disease no standard therapy exists in the second line or when patients are intolerant to the first line treatment. Therefore, treatment of these patients is usually following a very individual path in daily practice. Our aim was to retrospectively analyze treatment of patients with adrenocortical carcinoma in our tertiary center and compare treatment outcomes with reports in the literature. Our findings reflect the daily practice in adrenocortical carcinoma treatment and showed that surgery is the mainstay of therapy, even in some cases with metastatic disease. Adjuvant therapy in adrenocortical carcinoma was initiated less frequently than suggested by current guidelines. Furthermore, most of the patients in our cohort received more than one line of chemotherapy for metastatic or inoperable disease with overall survival rates comparable to those published. In summary, our analysis stresses the importance of clinical trial activity in this rare disease in order to standardize and improve adrenocortical carcinoma therapy more profoundly.
\end{abstract}

Key words: adrenocortical carcinoma, chemotherapy, mitotane, prognosis

Adrenocortical carcinoma (ACC) is a rare disease with an incidence of 2 cases per million people per year [1]. Surgery is the standard therapy for resectable disease although many patients are not eligible for surgery at diagnosis $[2,3]$ and approximately $80 \%$ of patients relapse after surgery [4]. The high frequency of disease recurrence, the substantial number of patients who are not resectable at baseline and the generally poor prognosis of patients with metastatic disease contribute to the overall devastating clinical outcome [5]. Undoubtedly there is a high medical need for improving ACC therapy, however little progress has been made over the past years to improve systemic treatment. Most clinical studies to date are either retrospective analyses or small phase II trials, which cannot provide level I medical evidence. To date, only a single randomized phase III trial in advanced ACC has been conducted so far [6]. No difference in overall survival was seen between the two treatment arms. However, combination chemotherapy with EDP-M (Etoposide, Doxoru- bicin, Cisplatin and Mitotane), showed a significantly better response rate (20\%) and progression free survival (5 months) than Sz-M (Streptozotocin and Mitotane) (8\% and 2.1 months) therefore considering EDP-M as the standard care the in first line in metastatic ACC. Although on average ACC patients have a dismal prognosis, reports suggest quite some variability for individual patients with regard to their clinical course. Overall survival of stage IV ACC can range from 9 to 25 months depending on a number of prognostic factors [7]. This heterogeneity seems to be even more evident in patients receiving mitotane therapy with a survival ranging from 2 to 190 months suggesting that systemic therapy can have an impact on survival [8]. Contributing factors for a variable outcome are the underlying biology of the disease and presumably the impact of treatment at least for some patients. In this retrospective analysis, we have evaluated clinical data, treatment and outcome in 49 patients with ACC treated in our center from 1996 to 2014. 


\section{Patients and methods}

Patients. Individual patient charts were retrieved from our clinical data bank at our tertiary center. Patients with adrenocortical carcinoma with either localized or advanced disease were included. Relevant clinical, laboratory and pathological data were collected. Diagnosis was established following the Weiss criteria used to diagnose adrenocortical neoplasm [9]. Resection margins were assessed from pathological reports and surgical reports. Radiological scans were reviewed when treatment response was not stated in the clinical charts. Response evaluation was performed according to RECIST 1.1 whenever possible [10]. Patients were either grouped as responders (stable disease or better) or non-responders (progressive disease). Resection margins were classified as R0 (resection margins free of microscopic disease), R1 (microscopic evidence for residual malignancy at the resection margin) or R2 (macroscopic positive margins). Overall survival was defined as the time between diagnosis and death. Progression-free survival was defined as the time between diagnosis and progression or death, or last visit for patients alive without progression. Time-to-progression is defined as the time between the start with chemotherapy and progression, or death or last visit for patients without progression. Time-to-progression was analyzed separately in two distinct

Table 1. Patient characteristics.

\begin{tabular}{|c|c|c|}
\hline T-stage & \multicolumn{2}{|c|}{ total $\mathrm{n}=45$} \\
\hline 1 & $\mathrm{n}=7$ & $15.6 \%$ \\
\hline 2 & $\mathrm{n}=13$ & $28.9 \%$ \\
\hline 3 & $\mathrm{n}=15$ & $33.3 \%$ \\
\hline 4 & $\mathrm{n}=10$ & $22.2 \%$ \\
\hline N-stage & \multicolumn{2}{|c|}{ total $n=30$} \\
\hline 0 & $\mathrm{n}=17$ & $56.7 \%$ \\
\hline 1 & $\mathrm{n}=13$ & $43.3 \%$ \\
\hline Variable & \multicolumn{2}{|c|}{ total $\mathrm{n}=49$} \\
\hline No metastasis & $\mathrm{n}=17$ & $34.7 \%$ \\
\hline Synchronous metastasis & total $n=19$ & $38.8 \%$ \\
\hline Metachronous metastasis & total $n=13$ & $26.5 \%$ \\
\hline \multicolumn{3}{|l|}{ Metastatic sites synchronous } \\
\hline Liver & $\mathrm{n}=11$ & $22.5 \%$ \\
\hline Lung & $\mathrm{n}=13$ & $26.5 \%$ \\
\hline Lymph nodes & $\mathrm{n}=9$ & $18.4 \%$ \\
\hline Skeletal & $\mathrm{n}=2$ & $4.1 \%$ \\
\hline Other (pleura, pancreas, CNS, colon, kidney) & $\mathrm{n}=5$ & $10.2 \%$ \\
\hline \multicolumn{3}{|l|}{ Metastatic site metachronous } \\
\hline Liver & $\mathrm{n}=16$ & $32.7 \%$ \\
\hline Lung & $\mathrm{n}=19$ & $38.8 \%$ \\
\hline Lymph nodes & $\mathrm{n}=12$ & $24.5 \%$ \\
\hline Skeletal & $\mathrm{n}=4$ & $8.2 \%$ \\
\hline $\begin{array}{l}\text { Other (pleura, pancreas, CNS, spleen, } \\
\text { stomach, muscle, peritoneal, colon, kidney) }\end{array}$ & $\mathrm{n}=13$ & $26.5 \%$ \\
\hline
\end{tabular}

Abbreviations: CNS; central nervous system. patient groups: patients receiving palliative chemotherapy and patients receiving mitotane in adjuvant setting.

Statistical analysis. Summary statistics are presented as frequencies and percentages for categorical variables. KaplanMeier estimates are used for estimating overall survival, progression-free survival or time-to-progression. Summary statistics for follow-up time are based on the Kaplan-Meier estimate of potential follow-up [11]. Cox regression models are used to analyze the association between prognostic factors and outcome. Considering the multivariable analysis, a stepwise selection procedure was applied for selecting a set variables as independent predictors of outcome (overall survival and progression-free survival). Results are presented as hazard ratios (HR) with 95\% confidence intervals (CI). Analyses were performed using SAS software (version 9.4 of the SAS System for Windows).

\section{Results}

Patient characteristics. A total of 49 patients were included in this study. The median age at diagnosis was 47 years (range: 3 to 81 years) and more female patients $(n=32 ; 65 \%)$ than male patients $(n=17 ; 35 \%)$ were identified. More tumors were located at the right adrenocortical gland $(25 / 49 ; 51 \%)$ than on the left side $(24 / 49 ; 49 \%)$. Hormonal excess was diagnosed in $29 \%$ of the cases. In $65 \%$ of the patients the tumor was classified as being non-functional. For a minority of the patients (6\%) the hormonal status was not investigated at initial presentation due to the lack of endocrine related symptoms. Most of the tumors were aldosterone (24\%) secreting. Cortisol (20\%) and androgen (14\%) excess was identified less frequently. The initial tumor stage is outlined in Table 1.

Follow up and treatment. Approximately, one third of the patients $(n=17 ; 34.6 \%)$ had no metastatic disease at presentation. Two thirds of the patients had metastatic disease occurring either synchronous $(38.8 \%)$ or metachronous $(26.5 \%)$. The most prevalent metastatic sites where liver, lung, lymph nodes and bone, however patients with metachronous metastasis more frequently presented with unusual metastatic localizations like pancreas, central nervous system or muscle metastasis. The median time to the occurrence of metachronous metastasis was 20 months (range: 3 to 61 months). The vast majority of the patients $(n=41 / 49)$ underwent surgical resection, with only 8 patients not being operated due to the extensive metastatic disease. Surgical resection was either with curative intent or with the aim of tumor debulking to gain better control of hormonal excess. A complete resection was achieved in 30/41 patients (75\%). In 8 out of 41 patients (20\%) surgery was repeated at the time of relapse. In 19 out of 41 patients $(46 \%)$ who underwent resection, surgery was the only treatment modality. For the remaining 22 patients (54\%) treatment with mitotane was initiated, either adjuvant $(n=13)$ or additive $(n=9)$ following incomplete resection. A total of 23 patients were disease free, either with surgery or surgery in combination with mitotane therapy. The majority of the 
Table 2. Chemotherapy protocols for palliative treatment $(n=22)$.

\begin{tabular}{lc}
\hline Chemotherapy & Number of patients \\
\hline Mitotane & 7 \\
Mitotane-EDP & 7 \\
CAP & 2 \\
Cisplatin/Etoposide & 4 \\
EDP & 2 \\
Lines of treatment & Number of patients \\
1 line & 7 \\
2 lines & 7 \\
3 lines & 8 \\
\hline
\end{tabular}

Abbreviations: CAP: cyclophosphamide adriamycin cisplatin, EDP: etoposide doxorubicin cisplatin.

patients who received adjuvant mitotane therapy relapsed $(n=9 / 13)$. A total of 22 patients in our cohort received palliative therapy either upfront $(\mathrm{n}=8)$ or at the time of relapse $(n=14)$. The chemotherapy protocols used are outlined in Table 2. A total of 7 patients received 1 line of palliative therapy (32\%), 7 patients received two lines of systemic treatment (32\%). Eight patients received more than 2 lines (36\%) with one patient even receiving 9 lines of different systemic therapy (5\%). The majority of patients received EDP-M chemotherapy in the first line and a 5FU based regimen in the second line. Protocols in the 3rd line or later were doxorubicine monotherapy $(\mathrm{n}=1)$, carboplatinum $(\mathrm{n}=1)$, gemcitabine/ xeloda $(n=1)$, carboplatinum/taxol $(n=1), 5 F U$ monotherapy $(\mathrm{n}=1)$ and sunitinib $(\mathrm{n}=1)$. Two patients were included in a phase I study with a PI3K inhibitor, which evaluated safety and pharmacokinetics of pilaralisib in patients with advanced solid tumors or relapsed/refractory lymphoma [12]. Unfortunately, none of the included patients showed an objective response. Best tumor response in patients receiving chemotherapy was stable disease according to RECIST 1.1.

Outcome. At last follow-up, 17 of 49 patients had died (35\%) and 32 patients were still alive. After a median followup of 3.6 years the median overall survival of all patients was 3.8 years with an estimated 5-years survival rate of $48 \%$ (95\% CI 29.5-64.7\%). For patients receiving mitotane in the adjuvant setting we saw a median progression free survival of 1.4 years (95\% CI: $0.33-2.9$ ) and a median overall survival of 2.9 years with a 5 year estimated overall survival rate of $39 \%$ (95\% CI: 12.6-65\%). Patients receiving palliative therapy had a median overall survival of 1.8 years with a median time to progression of 5 months (95\% CI: 3-10 months) following the first line therapy. Univariate analysis revealed a significant association of higher T stage (HR: 2.989; $\mathrm{p}=0.0009$ ), positive resection margins (HR: $3.883 ; \mathrm{p}=0.05$ ) and synchronous metastasized disease (HR: $2.326 ; \mathrm{p}=0.001$ ) with shorter overall survival (Figure 1). Synchronous metastatic disease was identified as the only independent risk factor for shorter overall survival (HR: 13.448; $\mathrm{p}=0.093$ ). No evidence was found for an association between overall survival and surgery at relapse $(0.199 ; \mathrm{p}=0.117)$, hormonal excess (HR:
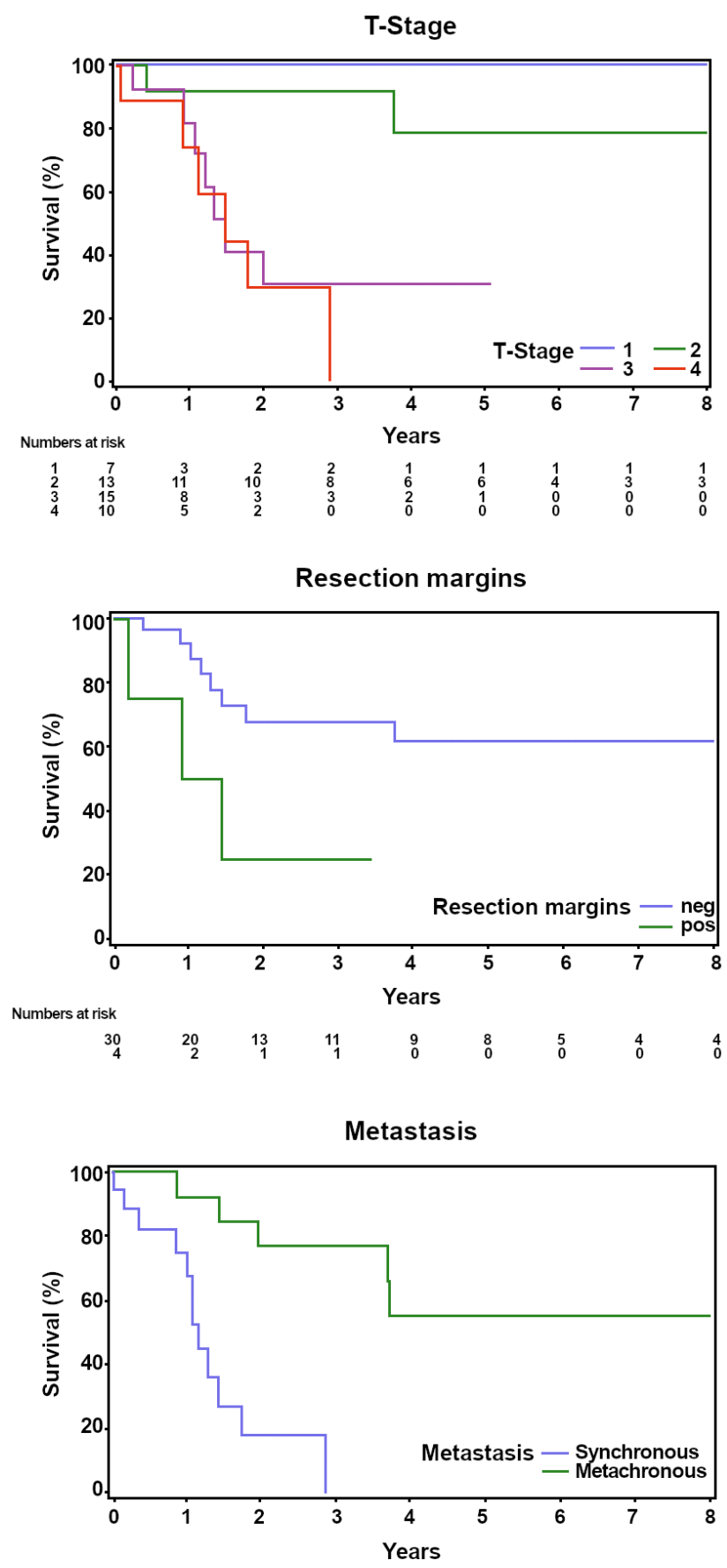

Numbers at risk

$\begin{array}{lllllllll}19 & 10 & 2 & 0 & 0 & 0 & 0 & 0 & 0 \\ 13 & 12 & 10 & 9 & 5 & 4 & 3 & 2 & 1\end{array}$

Figure 1. Kaplan Meier survival analysis. Kaplan Meier plot illustrating the correlation between overall survival and T-stage, resection margins or metastatic disease over time.

1.046; $\mathrm{p}=0.9381$ ), or nodal involvement (HR: $2.802 ; \mathrm{p}=0.078$ ) neither did we find evidence of an association between the use of chemotherapy and time to tumor progression or overall survival (HR: 2.284; $\mathrm{p}=0.107$ ). 


\section{Discussion}

ACC is considered not just an orphan disease but also a disease in which only few studies have been conducted in the past. As a result, the level of evidence for systemic treatment is very limited, creating considerable variability in treating these patients. Our retrospective analysis was conducted in order to describe the variability in treatment modalities in a tertiary medical center.

With regard to demographic data, our study cohort is comparable to previously published data, although we identified an equal distribution of affected sites and a lower incidence of hormonal excess [13-16]. Amongst our patients, only $29 \%$ of cases exhibited hormonal excess, compared to $42-76 \%$ in other reports $[13,15]$. This cannot be explained by reduced testing, as only in $6 \%$ did not undergo hormonal evaluation. In contrast to our study, previous work has suggested that hormonal hypersecretion is an independent risk factor for reduced overall survival $[13,15]$. The lower rate of functional tumors did not result in a better overall survival in our cohort compared to other cohorts, which might be attributed to the higher disease stage at diagnosis seen in our cohort. The fact that non-functional tumors can grow considerable in size before becoming symptomatic would explain this correlation. In contrast, hormonal excess usually enables earlier diagnosis due to its clinical signs. Most likely our cohort is subjected to a referral bias typically seen in tertiary health care centers. Bigger tumors pose a surgical challenge requiring experience and often the full panel of multimodal treatment strategies, something smaller health care providers might not be able to provide.

The number of patients undergoing surgical resection is high in patients with ACC. In our series $83 \%$ of the patients received surgery, which is comparable to results reported by other groups, with resection rates varying from 80 to $90 \%$ $[13,15]$. Most of the patients received a complete resection of the primary tumor, with resection of metastasis when complete resection was deemed feasible by the surgeon. In $26 \%$ of the patients a $\mathrm{R} 1$ or $\mathrm{R} 2$ resection was performed, which is comparable to previous reports [16]. Some centers advocate even surgical resection at relapse as this might improve survival, although sound evidence is lacking to advocate such measures $[2,17,18]$. The number of patients undergoing re-resection is variable with more than $40 \%$ in one series [2] and only $14 \%$ in another [17]. In our series the rate of surgery after relapse was rather low (20\%) and median overall survival in these patients showed considerable variability ranging from 7 to 84 months (median 49 months), suggesting that some patients might benefit from surgery at relapse. However, due to the lack of prospective randomized studies such an approach cannot be considered as standard of care in patients with ACC.

Mitotane is the only drug accepted as an adjuvant therapy in resected ACC. This is based on sporadic reports and retrospective studies and is therefore associated with a low level of evidence $[19,20]$. In our cohort adjuvant treatment was initiated in $31 \%$ of the patients, whereas additive treatment was given in $21 \%$ of cases in the setting of measurable, non-progressing disease after surgery. The number of patients treated at our center with adjuvant therapy is lower compared to other centers, where almost $70 \%$ of patients received adjuvant mitotane $[13,15]$. We initiated adjuvant mitotane in patients with T2 stage or higher, of which more than $50 \%$ had stage T3 or T4 disease. However, still $44 \%$ of patients in stage T3 and T4 did not receive adjuvant therapy. The overall survival of adjuvant treated patients was 2.9 years, which is lower than 9.1 years reported by Terzolo et al. [21] or 5.3 years reported by Grubbs et al. [19]. However, a huge variability in terms of overall survival following adjuvant therapy with mitotane can be noted, which is most likely due to the retrospective nature of most analyses. In addition, most of our patients receiving adjuvant mitotane had stage III or stage IV disease, as opposed to patients in larger series, where more than $50 \%$ of the patients had stage I or II. This could possibly be attributed to referral bias, as patients with more extensive disease or non-typical metastatic sites are more likely to be referred to a tertiary center. Indeed, more patients with stage III or IV disease were referred to us $(n=24)$ compared to stage I or II $(n=11)$, however this proved to be of no statistical significance $(\mathrm{p}=0.095)$. The lack of a high level of evidence, the uncertainty with regard to duration of adjuvant treatment and the poor tolerability in therapeutic dose ranges might be reasons why fewer patients were treated with stage III or IV disease, compared to the guidelines or reports from literature.

For patients receiving palliative chemotherapy the progression free survival was 5 months (range 3 to 10 months), which is in accordance with the median PFS seen in patients treated in the FIRM-ACT trial [6]. Despite the fact that EDP-mitotane is considered the standard of care in unresectable or metastatic ACC, only 7 patients received this combination as the first line therapy. Another 7 patients were treated with mitotane monotherapy. The main reasons for not choosing combination therapy instead of monotherapy were reduced performance status of the patients, concerns about toxicity and the fact that patients were treated before results of the FIRM-ACT study were publicly available. Although there is no standard of care in regard to the second line treatment, more than half of the patients actually received two or more lines of systemic therapy. Two of the patients in our cohort even entered a phase I clinical trial. This illustrates that trials in the second line would be feasible and worthwhile to be conducted and that there is an unmet medical need for this population of patients. For the time being, EDP-mitotane is considered as the first line standard of care in patients fit enough to undergo combination therapy with 4 drugs. Alternatively, Cisplatinum-Etoposide or CAP can be used for patients intolerant to mitotane or with contraindications for one or the other drug. In addition, 5FU based chemotherapy protocols have been used. However as mentioned previously, 
scientific evidence concerning the second and the third line therapies are scarce. Although clinical trials are evidently difficult to conduct, owing to the rarity of the disease, several examples in recent years exist, showing that for rare and even ultra-rare diseases clinical trials have been conducted in a multinational effort.

Our single center analysis shows that patients with ACC are treated in a multidisciplinary approach. Our data reflecting a real-life setting, show that surgery is not only performed in a curative intent but also in order to minimize tumor load. Systemic therapy lacks standardization making it difficult to interpret overall survival data. Since patients are usually receiving more than one line of therapy, more clinical trial activity beyond the first line therapy is desperately needed in order to improve standardization as well as treatment options and prognosis in patients with ACC.

\section{References}

[1] ROMAN S. Adrenocortical carcinoma. Curr Opin Oncol 2006; 18: 36-42. https://doi.org/10.1097/01. cco.0000198976.43992.14

[2] SCHULICK RD, BRENNAN MF. Long-term survival after complete resection and repeat resection in patients with adrenocortical carcinoma. Ann Surg Oncol 1999; 6: 719-726. https://doi.org/10.1007/s10434-999-0719-7

[3] POMMIER RF, BRENNAN MF. An eleven-year experience with adrenocortical carcinoma. Surgery 1992; 112: 963-970.

[4] STOJADINOVIC A, GHOSSEIN RA, HOOS A, NISSAN A, MARSHALL D et al. Adrenocortical carcinoma: clinical, morphologic, and molecular characterization. J Clin Oncol 2002; 20: 941-950. https://doi.org/10.1200/ JCO.2002.20.4.941

[5] FASSNACHT M, JOHANSSEN S, QUINKLER M, BUCSKY P, WILLENBERG HS et al. Limited prognostic value of the 2004 International Union Against Cancer staging classification for adrenocortical carcinoma: proposal for a Revised TNM Classification. Cancer 2009; 115: 243-250. https://doi. org/10.1002/cncr.24030

[6] FASSNACHT M, TERZOLOL M, ALLOLIO B, BAUDIN E, HAAK $\mathrm{H}$ et al. Combination chemotherapy in advanced adrenocortical carcinoma. N Engl J Med 2012; 366: 2189-2197. https://doi.org/10.1056/NEJMoa1200966

[7] ASSIE G, ANTONI G, TISSIER F, CAILLOU B, ABIVEN G et al. Prognostic parameters of metastatic adrenocortical carcinoma. J Clin Endocrinol Metab 2007; 92: 148-154. https:// doi.org/10.1210/jc.2006-0706

[8] BAUDIN E, LEBOULLEUX S, AL GHUZLAN A, CHOUGNET C, YOUNG J et al. Therapeutic management of advanced adrenocortical carcinoma: what do we know in 2011? Horm Cancer 2011; 2: 363-371. https://doi.org/10.1007/ s12672-011-0094-2

[9] WEISS LM. Comparative histologic study of 43 metastasizing and nonmetastasizing adrenocortical tumors. Am J Surg Pathol 1984; 8: 163-169. https://doi.org/10.1097/00000478198403000-00001
[10] EISENHAUER EA, THERASSE P, BOGAERTS J, SCHWARTZ LH, SARGENT D et al. New response evaluation criteria in solid tumours: revised RECIST guideline (version 1.1) Eur J Cancer 2009; 45: 228-247. https://doi. org/10.1016/j.ejca.2008.10.026

[11. SCHEMPER M, SMITH TL. A note on quantifying followup in studies of failure time. Control Clin Trials 1996 17: 343-346. https://doi.org/10.1016/0197-2456(96)00075-x

[12] BECHTER OE, DUMEZ H, COSTERMANS J, PUNIE K, HSU K et al. Phase I safety and pharmacokinetic dose-escalation study of pilaralisib polymorph E, a phosphoinositide 3-kinase inhibitor in tablet formulation, in patients with solid tumors or lymphoma. Cancer Chemother Pharmacol 2016; 78: 83-90. https://doi.org/10.1007/s00280-016-3056-0

[13] AYALA-RAMIREZ M, JASIM S, FENG L, EJAZ S, DENIZ F et al. Adrenocortical carcinoma: clinical outcomes and prognosis of 330 patients at a tertiary care center. Eur J Endocrinol 2013; 169: 891-899. https://doi.org/10.1530/EJE-130519

[14] GRATIAN L, PURA J, DINAN M, REED S, SCHERI R et al. Treatment patterns and outcomes for patients with adrenocortical carcinoma associated with hospital case volume in the United States. Ann Surg Oncol 2014; 21: 3509-3514. https://doi.org/10.1245/s10434-014-3931-z

[15] ABIVEN G, COSTE J, GROUSSIN L, ANRACT P, TISSIER $F$ et al. Clinical and biological features in the prognosis of adrenocortical cancer: poor outcome of cortisol-secreting tumors in a series of 202 consecutive patients. J Clin Endocrinol Metab 2006; 91: 2650-2655. https://doi.org/10.1210/ jc. 2005-2730

[16] BILIMORIA KY, SHEN WT, ELARAJ D, BENTREM DJ, WINCHESTER DJ et al. Adrenocortical carcinoma in the United States: treatment utilization and prognostic factors. Cancer 2008; 113: 3130-3136. https://doi.org/10.1002/ cncr.23886

[17] BELLANTRONE R, FERRANTE A, BOSCHERINI M, LOMBARDI CP, CRUCITTI $P$ et al. Role of reoperation in recurrence of adrenal cortical carcinoma: results from 188 cases collected in the Italian National Registry for Adrenal Cortical Carcinoma. Surgery 1997; 122: 1212-1218. https:// doi.org/10.1016/s0039-6060(97)90229-4

[18] GAUJOUX S, AL-AHMADIE H, ALLEN PJ, GONEN M, SHIA J et al. Resection of adrenocortical carcinoma liver metastasis: is it justified? Ann Surg Oncol 2012; 19: 2643-2651. https://doi.org/10.1245/s10434-012-2358-7

[19] GRUBBS EG, CALLENDER GG, XING Y, PERRIER ND, EVANS DB et al. Recurrence of adrenal cortical carcinoma following resection: surgery alone can achieve results equal to surgery plus mitotane. Ann Surg Oncol 2010; 17: 263-270. https://doi.org/10.1245/s10434-009-0716-x

[20] TERZOLO M, BAUDING AE, ARDITO A, KROISS M, LEBOULLEUX $S$ et al. Mitotane levels predict the outcome of patients with adrenocortical carcinoma treated adjuvantly following radical resection. Eur J Endocrinol 2013; 169:263-270.

[21] TERZOLO M, ANGELI A, FASSNACHT M, DAFFARA F, TAUCHMANOVA L et al. Adjuvant mitotane treatment for adrenocortical carcinoma. N Engl J Med 2007; 356: 23722380. https://doi.org/10.1056/NEJMoa063360 